\title{
INCIDENCIA Y FACTORES DE RIESGO ASOCIADOS AL ÓBITO FETAL EN 2 HOSPITALES VENEZOLANOS
}

\author{
Erika Rojas ${ }^{1}$, Karimar Salas ${ }^{1}$, Gustavo Oviedo², Geovanny Plenzyk ${ }^{1}$ \\ ${ }^{1}$ Hospital Universitario Dr Ángel Larralde; ${ }^{2}$ Departamento de Salud Pública, Universidad de Carabobo, Valencia, Vene- \\ zuela.
}

\section{RESUMEN}

Objetivo: Evaluar la incidencia y factores de riesgo de óbito fetal en una población de embarazadas venezolanas. Metodología: estudio efectuado en 100 mujeres con diagnóstico de óbito fetal que acudieron al servicio de emergencia obstétrica de dos hospitales públicos en Valencia, Venezuela, en 2003. Resultados: Hubo 11.306 pérdidas fetales, 356 casos de óbito fetal y una tasa de mortalidad fetal tardía de 16,5/ 1000 nacidos vivos. De las embarazadas estudiadas $23 \%$ tenía más de 35 años de edad; $55 \%$ presentó muerte fetal posterior a la semana 28 de gestación; 31\% fumó durante el embarazo; $48 \%$ presentaron infecciones maternas y $38 \%$ hipertensión arterial. Las causas fetales y ovulares constituyeron el 39 y $61 \%$, respectivamente. Conclusiones: El óbito fetal es una complicación del embarazo frecuente y prevenible. Los principales factores de riesgo en este estudio fueron: infecciones maternas, hipertensión arterial y consumo de tabaco.

\section{PALABRAS CLAVES: Óbito fetal, mortalidad fetal tardía, estadísticas vitales, Venezuela}

\section{SUMMARY}

Objective: To evaluate the incidence and late fetal death risk factors. Methodology: A study was made in 100 women with a diagnosis of late fetal death assisted in two public hospitals in Valencia, Venezuela, during 2003. Results: There were 11,306 fetal losses, 356 cases of fetal death, with a late fetal mortality rate of $16.5 / 1000$ live birth; $23 \%$ of pregnant women were older than 35 years old; $55 \%$ had fetal death after the 28th week of gestation, $31 \%$ smoked during pregnancy; $48 \%$ of the women had infections, and $38 \%$ had high blood pressure. The fetal and ovular causes were $31 \%$ and $69 \%$, respectively. Conclusions: The fetal death is a potentially prevented complication and its main risk factors were: maternal infections, high blood pressure and smoking cigarettes.

\section{KEY WORDS: Fetal death, late fetal mortality, vital statistics, Venezuela}

\section{INTRODUCCIÓN}

La mujer embarazada y el feto, a pesar de cumplir con un proceso fisiológico, están expuestos a una serie de riesgos que amenazan su salud y vida (1). Entre las complicaciones del embarazo más temidas se encuentra el óbito fetal, llamado también muerte fetal tardía, si ocurre entre las 28 semanas de gestación y el parto. Se define muerte fetal intrauterina, el cese de la vida fetal a partir de las 20 semanas de gestación y con un peso mayor a 500 gramos $(2,3,4,5,6)$.

La etiopatogenia del óbito fetal es controversial, debido a que hay muchas causas relacionadas, las cuales se clasifican en maternas, fetales y ovulares. Entre los principales factores asociados 
a la muerte fetal están la enfermedad hipertensiva del embarazo, infección urinaria, diabetes mellitus, lupus eritematoso sistémico, edad materna superior a 35 años o menor a 20 años, nuliparidad, pérdidas fetales anteriores, anomalías cromosómicas fetales, tabaquismo, falta de control prenatal y traumatismos, entre otros $(7,8,9)$.

En Venezuela, para el año 1999, la tasa de mortalidad fetal tardía fue de 11,9 por 1000 nacidos vivos; cifras que pudieran considerarse elevadas al compararlas con otros países tales como Chile, México, Argentina y Estados Unidos (3, 6, $7,10,11)$. Sin embargo, según datos reportados por Faneite y cols (5), en algunas ciudades de Venezuela las cifras son aún mayores, tal es el caso del Hospital Adolfo Prince Lara de Puerto Cabello, Estado de Carabobo, donde la tasa de mortalidad fetal tardía fue de 20,56 por 1000 nacidos vivos.

Tomando en consideración esta situación se realizó la presente investigación, que tiene como objetivo la determinación de la incidencia y los factores asociados a la etiopatogenia del óbito fetal en embarazadas que acuden al Servicio de Obstetricia y Ginecología del Hospital Universitario "Dr Ángel Larralde" y a la Ciudad Hospitalaria "Dr Enrique Tejera", Valencia, Venezuela.

\section{MATERIAL Y METODOS}

Estudio descriptivo, no experimental, de tipo transversal, en embarazadas que acuden a la emergencia del servicio de Ginecología y Obstetricia del Hospital Universitario "Dr Ángel Larralde" y a la Ciudad Hospitalaria "Dr Enrique Tejera", de la ciudad de Valencia, Venezuela. Se estudiaron los casos de óbito fetal ingresados desde enero a diciembre de 2003, tomando una muestra al azar de 100 pacientes. Los datos se recolectaron mediante una entrevista y la revisión de la historia médica, tomando en cuenta las siguientes variables: edad cronológica y edad gestacional de la paciente, presencia de patologías maternas (infecciosas, hipertensión arterial, diabetes, renales, hematológicas, traumáticas), consumo de sustancias nocivas (tabaco, alcohol y drogas), defectos ovulares o placentarios y anomalías fetales. La información obtenida se presenta en tablas de distribución de frecuencia absoluta y relativa.

\section{RESULTADOS}

De las 32.850 pacientes que asistieron a la emergencia en los hospitales estudiados, 11.306 fueron pérdidas fetales, dentro de las que se encuentran 356 casos de óbito fetal, el resto son pérdidas fetales tempranas o abortos. Resultando una tasa de 16,52 muertes fetales tardías por cada 1000 nacidos vivos. Al analizar la incidencia de óbito fetal según grupos etarios (Tabla I) observamos que la mayor frecuencia correspondió a las embarazadas $\geq 35$ años de edad (23\%), seguido por el grupo entre 21 y 25 años (22\%). La edad gestacional mas frecuente en la cual ocurrió el óbito fetal fue en los mayores de 28 semanas (55\%). En cuanto al consumo de sustancias nocivas durante el embarazo, $31 \%$ de las pacientes fumaron cigarrillos y de ellas $21 \%$ lo hizo con frecuencia. De las 14 mujeres que consumían alcohol, sólo 4 lo hacían con frecuencia; mientras que de las 7 que consumieron drogas alucinógenas, 2 lo hicieron frecuentemente (Tabla II). Con respecto a los antecedentes patológicos (Tabla III), $48 \%$ presentó infección génito-urinaria y $38 \%$ hipertensión arterial durante el embarazo. Al analizar la etiología del óbito, 39\% fueron por defectos fetales, de los cuales $22 \%$ eran por displasias o disrupciones; $61 \%$ fue por defectos ovulares y de ellos en $18 \%$ hubo ruptura prematura de membranas y en $16 \%$ abruptio placentae (Tabla IV).

\section{DISCUSIÓN}

La embarazada y el feto están expuestos a una serie de riesgos que amenazan su salud y su vida, por lo que no todos los embarazos tienen como resultado el nacimiento de un niño vivo y

Tabla I

INCIDENCIA DE ÓBITO FETAL SEGÚN GRUPO DE EDAD MATERNA Y EDAD GESTACIONAL

\begin{tabular}{lrr}
\hline Edad (años) & $n$ & $\%$ \\
\hline$<15$ & 5 & 5 \\
$16-20$ & 15 & 15 \\
$21-25$ & 22 & 22 \\
$26-30$ & 18 & 18 \\
$31-35$ & 17 & 17 \\
$>35$ & 23 & 23 \\
\hline Total & 100 & 100 \\
\hline Edad gestacional (semanas) & $n$ & $\%$ \\
\hline $20-28$ semanas & 29 & 29 \\
$>28$ semanas & 55 & 55 \\
Desconocida & 16 & 16 \\
\hline Total & 100 & 100 \\
\hline
\end{tabular}


Tabla II

\section{CONSUMO DE SUSTANCIAS NOCIVAS DURANTE EL EMBARAZO}

\begin{tabular}{lccr}
\hline Sustancia & Ocasional & Frecuente & $\%$ \\
\hline Cigarrillo & 10 & 21 & 31 \\
Alcohol & 10 & 4 & 14 \\
Drogas & 6 & 2 & 7
\end{tabular}

sano. De hecho, de los embarazos clínicamente reconocibles, un 10 a $15 \%$ se interrumpen espontáneamente $(12,13)$. Los resultados de la presente investigación demuestran que en los hospitales estudiados hubo una elevada tasa de mortalidad fetal tardía, alcanzando una cifra de 16,5 por cada 1000 nacidos vivos, si lo comparamos con las tasas de países latinoamericanos como Chile, Argentina y México donde la tasa es menor a 8/1000 nacidos vivos $(6,11)$. Sin embargo, los resultados son menores a lo encontrado por Faneite y cols, en el Hospital Adolfo Prince Lara de Puerto CabeIlo, Venezuela, reportando una tasa de 20,56 muertes fetales tardías por cada 1000 nacidos vivos entre los años 1997 y 2002 (5).

El factor edad es uno de los determinantes en la salud fetal, donde cerca del $10 \%$ de las mujeres con edades entre 20 y 30 años presentan muerte fetal intrauterina, incrementándose el riesgo de óbito a medida que avanza la edad (14). En estudios realizados por Carrillo y cols en Colombia, se encontró que la edad mas frecuente era en mayo-
Tabla III

PATOLOGÍAS MATERNAS PRESENTES AL MOMENTO DEL ÓBITO FETAL

\begin{tabular}{lrr}
\hline Patologías & $n$ & $\%$ \\
\hline Infecciones génito-urinarias & 48 & 48 \\
Hipertensión Arterial & 38 & 38 \\
Diabetes & 13 & 13 \\
Renales & 10 & 10 \\
Hematológicas & 8 & 8 \\
Traumatismos & 5 & 5
\end{tabular}

res de 28 años (15). Rodríguez y Hernández encontraron una asociación significativa entre muerte fetal tardía y edades maternas menores a 20 años y entre 30 y 34 años (16). Sin embargo, en otros estudios $(9,17)$, se encontró que la mayor frecuencia de óbito fetal estaba en madres con edad entre 25 y 29 años, pero con mayor riesgo en aquellas con edad superior a 35 años. En la presente investigación el $23 \%$ de las mujeres eran mayores de 35 años de edad, sin embargo el $20 \%$ tenían una edad menor a 20 años, lo cual significa que el riesgo del óbito fetal está presente no sólo en edades mayores sino también en la adolescencia. Nuestros resultados coinciden con la experiencia chilena de Donoso y cols quienes encontraron que las edades más frecuentes asociadas a la muerte fetal tardía son las correspondientes a madres jóvenes $\mathrm{o}$ a las mayores de 35 años $(18,19,20)$.

Tabla IV

ETIOLOGÍA DEL ÓBITO FETAL DE ACUERDO A DEFECTOS FETALES Y OVULARES

\begin{tabular}{llrr}
\hline & Alteración & $n$ & $\%$ \\
\hline \multirow{3}{*}{ Defectos fetales } & Displasias/disrupciones & 22 & 22 \\
& Malformaciones estructurales & 9 & 9 \\
& Malformaciones congénitas & 8 & 89 \\
\hline & Sub-total & 39 & 18 \\
& Ruptura prematura de membranas & 18 & 16 \\
& Abruptio placentae & 16 & 10 \\
Defectos ovulares & Circular del cordón & 10 & 6 \\
& Inserción baja de placenta & 6 & 4 \\
& Compresiones del cordón & 4 & 4 \\
& Nudo del cordón & 4 & 3 \\
\hline & Infarto placentario & 3 & 61 \\
\hline Total & Sub-total & 61 & 100 \\
\hline
\end{tabular}


Algunos estudios mencionan que la nicotina produce una vasculitis espástica uterina, ocasionando insuficiencia placentaria y retardo del crecimiento intrauterino, lo cual se ha asociado con desprendimiento prematuro de placenta (abruptio placentae) y muerte fetal $(2,3,9,21)$. El alcohol, por otro lado, puede causar una restricción del crecimiento intrauterino, malformaciones y muerte fetal (21). Adicionalmente se ha encontrado que las mujeres con un consumo crónico de alcohol y drogas alucinógenas, tienen baja ingesta de energía y nutrientes, ocasionando desnutrición materna, síndrome anémico, retardo del crecimiento fetal intrauterino, malformaciones fetales, insuficiencia placentaria y desprendimiento prematuro de placenta (21). En el presente estudio se observó un elevado consumo de sustancias tóxicas, en 21 de las pacientes que fumaban cigarrillos, en 4 de las que tomaban alcohol y 2 de las que consumían drogas alucinógenas. Similares resultados encontraron Rodríguez y Hernández (16) en Cuba, donde hubo asociación significativa entre el hábito de fumar y la muerte fetal tardía, con un riesgo relativo de 1,3 . Por otra parte en un trabajo realizado por Carrillo y cols, las mujeres que refirieron tabaquismo, presentaron 6,5 veces más posibilidades de tener óbito que las mujeres que no fumaban (15).

El embarazo es un estado de supresión inmunitaria, en donde las mujeres están propensas a infecciones, sobre todo genitourinarias, debido a cambios hormonales que propician modificaciones celulares y de $\mathrm{pH}(3,14,22)$. La infección de las vías urinarias es uno de los procesos mas frecuentes, diversas modificaciones anatómicas y fisiológicas parecen predisponer a esta situación, entre ellas la dilatación ureteropélvica (hidroureteronefrosis fisiológica), producto de la inhibición del tono ureteral por acción de la progesterona y prostaglandinas, y la obstrucción parcial del uréter por el útero grávido y rotado (22). En el presente estudio $48 \%$ de las pacientes presentó infecciones genitourinarias, mientras que en la investigación realizada por Sabalvarro (21) encontró que 36,1\% de las mujeres con óbito fetal tenían infecciones urinarias; estos procesos si no son tratados en su inicio pudieran ocasionar un proceso séptico que ocasionaría la muerte fetal.

La enfermedad hipertensiva es una de las complicaciones médicas más frecuentes del embarazo, la cual trae como consecuencia un deterioro de la función placentaria, disminución del aporte sanguíneo materno y desprendimiento prematuro placentario, incrementando así el riesgo de morta- lidad perinatal y sobre todo fetal $(21,22)$. Algunas investigaciones reportan que la hipertensión arterial (HTA) complica uno de cada diez embarazos y es la primera causa de morbimortalidad materna y fetal $(8,12,14)$. Aquino y cols, reportan que $41,9 \%$ de las madres con óbito fetal tuvieron HTA durante el embarazo, muy similar a lo referido por Sabalvarro en Nicaragua, donde el $40 \%$ de las madres eran hipertensas (21); mientras que Faneite y cols (5) en estudios realizados en Venezuela, encontraron que $30,4 \%$ de las pacientes con óbito fetal tuvieron HTA durante la gestación. En la presente investigación $38 \%$ de las pacientes presentaron HTA; situación que indica una alta incidencia de una patología prevenible o que puede ser tratada en épocas tempranas del embarazo disminuyendo el riesgo de una repercusión fatal en el feto.

Según algunos estudios, son muy diversos los factores asociados a óbito fetal; correspondiendo a factores maternos cerca (27\%), fetales (16\%), ovulares $(42 \%)$, y la deficiencia en la relación madre-feto $(16 \%)(2,14,23)$. Igualmente dichas investigaciones reportan que las causas más frecuentes para ocasionar un óbito debido a anomalías ovulares son las de origen placentario $(75 \%)$, seguidas por anomalías del cordón umbilical (25\%) (14). En la investigación realizada $39 \%$ de la incidencia fue atribuida a defectos fetales, en donde se observaron 5 casos de anencefalia, demostrando que las malformaciones estructurales del feto son causa importante de óbito; en cuanto a defectos ovulares, el $61 \%$ de las pacientes presentó patología de este tipo, encontrando entre las principales la ruptura prematura de membranas y el desprendimiento prematuro de placenta normoinserta. Estos resultados coinciden con la investigación realizada por Nurdan y cols en Brasil, por Sabalvarro en Nicaragua, Faneite y cols en Venezuela, donde el desprendimiento prematuro de placenta normoinserta fue una de las causas mas frecuentes de óbito fetal $(5,8,21)$. En cuanto a los defectos del cordón en esta investigación suman hasta un $18 \%$, resultados muy superiores a lo reportado por Faneite y cols con 8,28\% (5).

\section{CONCLUSIONES}

Se concluye que el óbito fetal puede presentarse a cualquier edad materna, sin embargo, el riesgo es mayor en edades menores a 20 años y superiores a 35 años. Entre los principales factores asociados están las infecciones genitourinarias de la madre, la hipertensión arterial inducida por el 
embarazo y el hábito tabáquico. Predominan las causas ovulares sobre las fetales. Un adecuado control prenatal permitirá identificar los factores de riesgos asociados a muerte fetal tardía, para así iniciar oportunamente un tratamiento destinado a evitar esa complicación.

\section{BIBLIOGRAFÍA}

1. Moore K. Embriología Clínica. Editorial McGraw Hill Interamericana, 2000; 85-195.

2. Uranga F, Uranga F. Obstetricia Práctica. Editorial Intermédica, 1974; 230-49.

3. Cunningham G, MacDonald P, Grant N, Leveno K, Gilstrap L, Hankins G, Clark S. Obstetricia. Editorial Panamericana, 1998; 235-45.

4. Poblete A, Donoso E. Definiciones e Indicadores Estadísticos de uso frecuente en Salud Pública Perinatal Nacional. Chile. (Documento en línea). Hallado en: http://www.cedip.cl/Guias/Salud\%20publica\%20 perinatal.htm.

5. Faneite P, González M, Faneite J, Meneses W, Álvarez L, Linares M, Rivera C. Actualidad en mortalidad fetal. Rev Obstet Ginecol Venez 2004; 64(2): 77-82.

6. Organización Panamericana de la Salud. Centro Latinoamericano de Perinatología y Desarrollo Humano. Mortalidad fetal, neonatal y perinatal. Actualización: mayo 2003. (Documento en línea). Hallado en: www.clap.ops-oms.org

7. Usandizaga J, De la Fuente P. Tratado de Ginecología y Obstetricia. (Vol I). Editorial Mc. Graw Hill Interamericana, 1997; 237-457.

8. Nurdan N, Mattar R, Camano L. Óbito fetal em microrregião de Minas Gerais: causas e fatores associados. Rev Bras Ginecol Obstet 2003; 25(2): 103-7.

9. Aquino M, Cecatti J, Mariani N. Risk factors associated to fetal death. São Paulo Med J 1998; 116: 1852-7.

10. Organización Panamericana de la Salud/Organización Mundial de la Salud. Análisis preliminar de la situación de salud en Venezuela. [Documento en línea]. Hallado en: www.ops-oms.org.ve/site/ venezuela/ven-sit-salud-core.htm-27k.

11. Donoso E. Desigualdad en mortalidad perinatal en- tre las comunas de la Provincia de Santiago. Rev Chil Obstet Ginecol 2004; 69(2): 112-7.

12. Gabbe S, Niebyl J, Simpson J. Obstetricia: normalidades y complicaciones en el embarazo. Editorial Marban, 2000; 307-27.

13. Grisolía P. Signos clínicos de alarma. Donosita. 1999. [Documento en línea]. Disponible: http:// 216.239.39.100/search?q=cache:q5T2eXH_gp8C: www. svnp. es / Documensignosdealarma.pdf+ Malformaciones+estructurales\&hl=es\&lr=lang_es \&ie=UTF-8.

14. Aller J, Pagés G. Obstetricia Moderna. Editorial McGraw Hill Interamericana 1999; 227-40.

15. Carrillo M, Rocha M, Contreras D, Sánchez M, Bermúdez M, Soto A, Sarmiento A. Factores asociados a los óbitos fetales. [Documento en línea]. Hallado en: http://www.encolombia.com/medicina/ginecologia/ obstetricia51400resumenes3.

16. Rodríguez A, Hernández I. Factores que inciden en la mortalidad fetal tardía. Rev Cubana Obstet Ginecol 2004; 30(2): Disponible en: http://scielo.sld.cu.

17. Vardanega K, Soares D, Paloshi W, Zapparoli M. Fatores de Risco para Natimortalidade em um Hospital Universitário da Região Sul do Brasil. Rev Bras Ginecol Obstet 2002; 24(9): 617-22.

18. Donoso E, Oyarzún E, Villarroel L. Mortalidad fetal tardía: Chile 1990-1996. Rev Chil OBstet Ginecol 1999; 64(1): 21-28.

19. Donoso E, Becker J, Villarroel L. Natalidad y riesgo reproductivo en adolescentes de Chile. Rev Panam Salud Publica 2003; 14(1): 3-8.

20. Donoso E, Villarroel L. Edad materna avanzada y riesgo reproductivo. Rev Med Chile 2003; 130(1): 55-9.

21. Sabalvarro L. Factores de riesgo asociados a óbito fetal en pacientes que fueron atendidos en el Hospital Fernando Vélez Paiz de Nicaragua. Enero 2002 a Octubre 2004. [Documento en línea]. Disponible en: http://www.minsa.gob.ni/bns/Monografias/Full_ text/gineco_obstetricia/update/FACTORES\%20DE\% 20RIESGO\%20ASOCIADOS\%20\%20A\%20OBITO \%20FETAL.pdf.

22. Oyarzún E, Badía J. Alto riesgo obstétrico. (Documento en línea). Hallado en: http://escuela.med.puc.cl /Departamentos/Obstetricia/AltoRiesgo/ muerte_fetal int.html

23. Schwarcz R, Duverges C, Díaz A, Fescina R. Obstetricia. Editorial El Ateneo 1999; 244-47. 